\title{
Cherenkov-transition radiation in a waveguide partly filled with a resonance dispersion medium
}

\author{
Tatiana Yu. Alekhina, Andrey V. Tyukhtin, and Aleksandra A. Grigoreva \\ Radiophysics Department of St. Petersburg University, 1 Ulyanovskaya, St. Petersburg 198504, Russia
}

(Received 11 June 2015; published 30 September 2015)

\begin{abstract}
We analyze the electromagnetic field of a charged particle that uniformly moves in a circular waveguide and crosses a boundary between a dielectric medium, which possesses frequency dispersion of a resonant type, and a vacuum area. The investigation of the waveguide mode components is analytically and numerically performed. It is shown that Cherenkov radiation (CR) can penetrate through the boundary, and Cherenkov-transition radiation (CTR) can be excited in the vacuum region. The conditions for this effect are obtained. It is shown that the CTR can be composed of a single mode (compared with the CTR in a nondispersive dielectric, which is multimode). The amplitude of the CTR is comparable to the amplitude of $\mathrm{CR}$ in an extensive range of parameters. The considered effect can be used to generate intense quasimonochromatic radiation.
\end{abstract}

DOI: 10.1103/PhysRevSTAB.18.091302

PACS numbers: 41.90.+e, 41.60.Bq, 41.60.Dk

\section{INTRODUCTION}

An investigation of the electromagnetic fields of charged particles that move in a waveguide through the boundary between different media is interesting in regards to the development of new methods for the generation of electromagnetic radiation and the acceleration of charged particles.

Note that the case of the border between a vacuum and cold isotropic plasma was previously investigated $[1,2]$. In this case, only transition radiation (TR) is excited; Cherenkov radiation (CR) is absent because the plasma refractive index is less than 1 . The energetic characteristics of the radiation are analyzed in [1], and the field structure is examined in [2].

The problems with transversal boundaries between a dielectric and a vacuum in a waveguide were also investigated in a series of papers [3-7]. Note that CR can be generated in a dielectric compared with cold plasma. This radiation is transmitted through the boundary and reflected off the boundary. This reflected and transmitted radiation is referred to as Cherenkov-transition radiation (CTR). The case of a charge flying from the vacuum area of a waveguide into a dielectric area $[3,5]$ is important for the wakefield acceleration technique $[8,9]$. The case of the charge flying from a dielectric area into a vacuum area $[4,5]$ is interesting for the new perspective method of the generation of terahertz and gigahertz radiation [10-12].

Note that the radiation of the charges in the dielectric loaded waveguides with transversal boundaries was only analyzed for nondispersive dielectrics. However, the

Published by the American Physical Society under the terms of the Creative Commons Attribution 3.0 License. Further distribution of this work must maintain attribution to the author(s) and the published article's title, journal citation, and DOI. medium dispersion can radically influence particle radiation. We consider the case of a semi-infinite dielectric that possesses a resonant dispersion. The field of a charge in this unbounded resonant medium was analyzed in [13-15]. The case of a regular (i.e., homogeneous along its axis) waveguide filled with this medium was analyzed in [16-18]. As discussed in these papers, the role of resonant dispersion is essential in these problems.

The primary focus is the study of CTR in the vacuum area of the waveguide because this effect can be especially interesting for the development of techniques for the generation of terahertz and gigahertz radiation.

\section{ANALYTICAL INVESTIGATION: GENERAL ASPECTS}

The problem statement is as follows: a small charge particle $q$ uniformly moves with the velocity $\vec{V}=c \beta \vec{e}_{z}$ along the $z$-axis of a metal circular waveguide with the radius $a$ and intersects the border $(z=0)$ between two semi-infinite media (Fig. 1). The nonmagnetic homogeneous isotropic medium on the left from the boundary $(z<0)$ is described by the following frequency-dependent electric permittivity:

$$
\varepsilon_{1}=\varepsilon_{1}(\omega)=1+\frac{\omega_{\mathrm{p}}^{2}}{\omega_{\mathrm{r}}^{2}-\omega^{2}},
$$

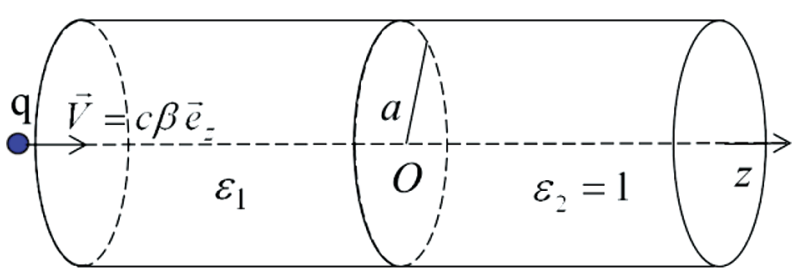

FIG. 1. Geometry of the problem. 
where $\omega_{\mathrm{p}}$ and $\omega_{\mathrm{r}}$ represent a plasma frequency and a resonant frequency, respectively. The permittivity at zero frequency is $\varepsilon_{1}=\varepsilon_{0}=1+\omega_{\mathrm{p}}^{2} / \omega_{\mathrm{r}}^{2}$, and $\varepsilon_{1} \rightarrow 1$ at $\omega \rightarrow \infty$. Note that Formula (1) is typical for different media in different states of aggregation of matter. Further we use, as an example, the ammonia which has resonance at $24 \mathrm{GHz}$. The medium on the right $(z>0)$ is a vacuum with $\varepsilon_{2}=1$. The charge intersects the boundary at the moment $t=0$.

The general analytical solution to the problem for two arbitrary isotropic homogeneous media is given in the form of a decomposition in infinite series of normal modes [1-7]. In this paper, we write only expressions for the magnetic field:

$$
\vec{H}_{1,2}=\vec{H}_{1,2}^{q}+\vec{H}_{1,2}^{b},
$$

where subscript 1 and subscript 2 relate to the areas $z<0$ and $z>0$, respectively. The first summand in (2) is the "forced" field, and the second summand in (2) is the "free" field (we employ terms from [19]). The forced field is the field of a charge in a regular waveguide. It is written in the following form:

$$
\begin{aligned}
H_{\varphi 1,2}^{q}= & \frac{2 q \beta^{2}}{\pi a^{2}} \sum_{n=1}^{\infty} \frac{\omega_{n} J_{1}\left(\chi_{n} r / a\right)}{J_{1}^{2}\left(\chi_{n}\right)} \\
& \times \int_{-\infty}^{+\infty} \frac{\exp \left\{\mathrm{i}\left[z(c \beta)^{-1}-\omega t\right]\right\}}{\omega^{2}\left(1-\beta^{2} \varepsilon_{1,2}\right)+\omega_{n}^{2}} d \omega,
\end{aligned}
$$

where $\omega_{n}=\chi_{n} c / a, \chi_{n}$ is the $n$th zero of the Bessel function $\left[J_{0}\left(\chi_{n}\right)=0\right]$.

The free field connected with the influence of the boundary is written in the form [3]

$$
\begin{aligned}
H_{\varphi 1,2}^{b}= & \frac{2 q \beta}{\pi c a^{2}} \sum_{n=1}^{\infty} \frac{\omega_{n} J_{1}\left(\chi_{n} r / a\right)}{J_{1}^{2}\left(\chi_{n}\right)} \\
& \times \int_{-\infty}^{+\infty} B_{n 1,2} \exp \left[\mathrm{i}\left(k_{\mathrm{zl}, 2}|z|-\omega t\right)\right] d \omega,
\end{aligned}
$$

where

$B_{n 1,2}=\frac{\left(\varepsilon_{1,2}-\varepsilon_{2,1}\right) \omega\left[\omega\left( \pm \varepsilon_{1,2} \beta^{2} \mp 1\right)-\beta \sqrt{\omega^{2} \varepsilon_{2,1}-\omega_{n}^{2}}\right]}{g(\omega)\left[\omega^{2}\left(1-\varepsilon_{1,2} \beta^{2}\right)+\beta^{2} \omega_{n}^{2}\right]\left(\omega \pm \beta \sqrt{\omega^{2} \varepsilon_{2,1}-\omega_{n}^{2}}\right)}$,

$g(\omega)=\varepsilon_{1} \sqrt{\omega^{2}-\omega_{n}^{2}}+\sqrt{\omega^{2} \varepsilon_{1}-\omega_{n}^{2}}$.

Here,

$$
k_{z 1,2}=\sqrt{\omega^{2} \varepsilon_{1,2}-\omega_{n}^{2}} / c,
$$

where $\operatorname{Im} k_{z 1,2}>0$ if the radicand is real negative or complex and $\operatorname{Re}\left(\omega k_{z 1,2}\right)>0$ if the radicand is real positive. (Note that we initially assume that a small imaginary part of the permittivity $\operatorname{Im} \varepsilon_{1,2}$ exists. However, it is only applied to determine the comparative disposition of the singularities and the integration pass. Subsequently, we tend this value to zero.)

We investigate expressions (3) and (4) for the case of the boundary between the resonant dielectric and the vacuum area. Note that the forced field was previously investigated [16-18]. Therefore, the main attention is focused on the free-field components (4). They are analyzed using two methods: an analytical method and a numerical one (they were developed in our papers [2-7] for different problems with sectionally homogeneous waveguides). In this paper, we only provide the most important results.

The analytical investigation is based on the complex variable function theory. The first step is a study of the integrand singularities in (4) on the complex plane of $\omega$ which are listed as follows:

(i) the branch points of the radical $k_{z 1}$

$$
\begin{aligned}
\pm \tilde{\omega}_{n}^{(1)}= & \pm \omega_{\mathrm{r}}\left\{\left[\varepsilon_{0}+y_{n}^{2}-\sqrt{\left(\varepsilon_{0}+y_{n}^{2}\right)^{2}-4 y_{n}^{2}}\right] / 2\right\}^{1 / 2}-\mathrm{i} 0 \\
\pm \tilde{\omega}_{n}^{(2)}= & \pm \omega_{\mathrm{r}}\left\{\left[\varepsilon_{0}+y_{n}^{2}+\sqrt{\left(\varepsilon_{0}+y_{n}^{2}\right)^{2}-4 y_{n}^{2}}\right] / 2\right\}^{1 / 2}-\mathrm{i} 0 \\
& \pm \omega_{\mathrm{r}}-\mathrm{i} 0 ;
\end{aligned}
$$

(ii) the branch points of the radical $k_{2}$

$$
\pm \omega_{n}-\mathrm{i} 0
$$

(iii) the poles

$$
\begin{aligned}
& \pm \omega_{0 n}^{(11)}= \pm \omega_{\mathrm{r}} x_{0 n}^{(11)}-\mathrm{i} 0, \quad \pm \omega_{0 n}^{(12)}= \pm \mathrm{i} \omega_{\mathrm{r}} x_{0 n}^{(12)}, \\
& \pm \omega_{0 n}^{(2)}= \pm \mathrm{i} \omega_{\mathrm{r}} x_{0 n}^{(2)}, \quad x_{0 n}^{(2)}=\beta y_{n}\left(1-\beta^{2}\right)^{-1 / 2} .
\end{aligned}
$$

Here,

$$
\begin{gathered}
y_{n}=\omega_{n} / \omega_{\mathrm{r}}=\chi_{n} / x_{\mathrm{r}}, \quad x_{\mathrm{r}}=a \omega_{\mathrm{r}} / c, \\
x_{0 n}^{(11)}=\left[S+1-\beta^{2}\left(\varepsilon_{0}+y_{n}^{2}\right)\right]^{1 / 2}\left(2-2 \beta^{2}\right)^{-1 / 2}, \\
x_{0 n}^{(12)}=\left[S-1+\beta^{2}\left(\varepsilon_{0}+y_{n}^{2}\right)\right]^{1 / 2}\left(2-2 \beta^{2}\right)^{-1 / 2}, \\
S=\sqrt{\left[1+\beta^{2}\left(\varepsilon_{0}+y_{n}^{2}\right)\right]^{2}+4 \beta^{2}\left(1-\beta^{2}\right) y_{n}^{2}} .
\end{gathered}
$$

Note that the singularities placed on the real axis are slightly shifted downward from the axis if small losses are taken into account (Fig. 2 shows the comparative disposition of an initial integration pass and the singularities). The branch cuts are defined by the following equations

$$
\operatorname{Re} \sqrt{\omega^{2} \varepsilon_{1}-\omega_{n}^{2}}=0, \quad \operatorname{Re} \sqrt{\omega^{2}-\omega_{n}^{2}}=0 .
$$

The initial integration path goes along the upper edge of the cuts.

Note that the poles $\pm \omega_{0 n}^{(11)}$ and $\pm \omega_{0 n}^{(12)}$ are also the singularities of the integrands in (3) for the forced field in medium 1 (left from the boundary). With the residue 


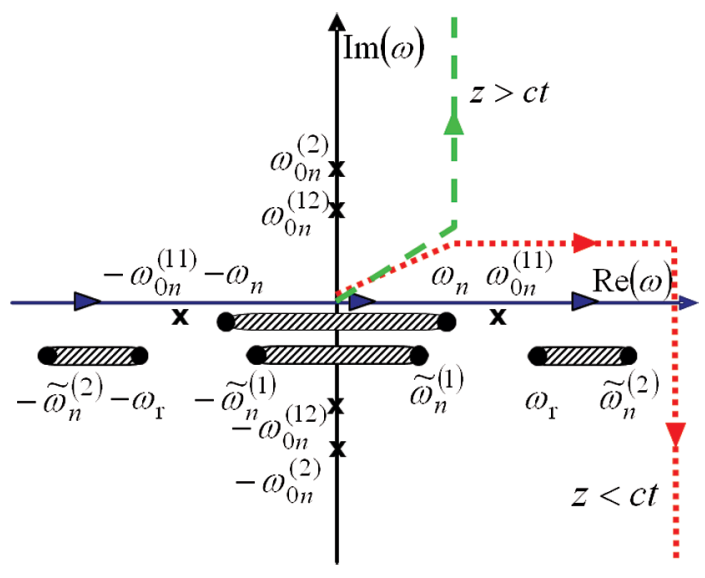

FIG. 2. Singularities, branch cuts, and integration paths in the complex plane of $\omega$ for the field components in a vacuum.

theorem, these poles yield the following expression for the magnetic component of the forced field:

$$
\begin{aligned}
H_{\varphi 1}^{q} & =\frac{q}{a^{2}} \sum_{n=1}^{\infty} \frac{\omega_{n} J_{1}\left(\chi_{n} r / a\right)}{J_{1}^{2}\left(\chi_{n}\right)}\left(I_{11}^{q}+I_{12}^{q}\right), \\
I_{11}^{q}= & A^{\mathrm{CR}} \sin \left[\omega_{0 n}^{(11)}\left(\frac{z}{c \beta}-t\right)\right] \theta(\beta c t-z), \\
A^{\mathrm{CR}}= & \frac{2 \sqrt{2} \beta^{2}}{\omega_{\mathrm{r}} \sqrt{1-\beta^{2}}} \frac{S-1-\beta^{2}\left(\varepsilon_{0}+y_{n}^{2}-2\right)}{S \sqrt{S+1-\beta^{2}\left(\varepsilon_{0}+y_{n}^{2}\right)}} \\
I_{12}^{q}= & \frac{\sqrt{2} \beta^{2}}{\omega_{\mathrm{r}} \sqrt{1-\beta^{2}}} \frac{S+1+\beta^{2}\left(\varepsilon_{0}+y_{n}^{2}-2\right)}{S \sqrt{S-1+\beta^{2}\left(\varepsilon_{0}+y_{n}^{2}\right)}} \\
& \times \exp \left(-\omega_{\mathrm{r}} x_{0 n}^{(12)}\left|\frac{z}{c \beta}-t\right|\right),
\end{aligned}
$$

where $\theta(x)$ is the Heaviside step function.

The forced field includes CR (9) if the condition $\varepsilon_{1}(\omega) \beta^{2}>1$ is fulfilled. One can see that the frequency of the radiated waves lays within the interval $\omega_{\mathrm{c}}<\omega<\omega_{\mathrm{r}}$, where $\omega_{\mathrm{c}}=\omega_{\mathrm{r}}\left(1-\varepsilon_{0} \beta^{2}\right)^{1 / 2}\left(1-\beta^{2}\right)^{-1 / 2}$ if $\varepsilon_{0} \beta^{2}<1$ and $\omega_{\mathrm{c}}=0$ if $\varepsilon_{0} \beta^{2}>1$. Thus, CR is generated in a medium with resonance dispersion for any velocity of the charge compared with nondispersive dielectrics, when the

generation only occurs if the charge velocity exceeds the Cherenkov threshold, that is, the condition $\varepsilon_{0} \beta^{2}>1$ is fulfilled. Note that the considered case can be reduced to the nondispersive dielectrics case with the permittivity $\varepsilon_{0}$ in the limiting process when $\omega_{\mathrm{r}} \rightarrow \infty, \omega_{\mathrm{p}} \rightarrow \infty$ and $\varepsilon_{1} \rightarrow \varepsilon_{0}$.

For the forced electric field, one more pair of poles $\pm \sqrt{\omega_{\mathrm{r}}^{2}+\omega_{\mathrm{p}}^{2}}$ contributes to the field and produces plasma oscillations. However, these poles give no significant contribution into the free field in the medium because they are always situated in the frequency range of nonpropagating waves $\omega_{\mathrm{r}}<\sqrt{\omega_{\mathrm{r}}^{2}+\omega_{\mathrm{p}}^{2}}<\tilde{\omega}_{n}^{(2)}$ (Fig. 2).

The forced field in the vacuum part of the waveguide has a quasi-Coulomb character. It is written in the form

$$
H_{\varphi 2}^{q}=\frac{2 q \beta}{a^{2} \sqrt{1-\beta^{2}}} \sum_{n=1}^{\infty} \frac{J_{1}\left(\chi_{n} r / a\right)}{J_{1}^{2}\left(\chi_{n}\right)} \exp \left[-\omega_{\mathrm{r}} x_{0 n}^{(2)}\left|\frac{z}{c \beta}-t\right|\right] .
$$

\section{ANALYTICAL INVESTIGATION: THE CTR}

Our main interest is an analysis of radiation in the vacuum area. To obtain asymptotic expressions for the freefield mode, we can use the steepest descend technique [20]. Note that the procedure for the vacuum area $(z>0)$ is similar to the procedure that was developed for different situations in our previous study [2-7].

The most important results of the analytical investigation for the free field in the area $z>0$ concern the contributions of the poles $\pm \omega_{0 n}^{(11)}$ described by Eq. (7). They give the transmitted wave of the CR or the CTR in the vacuum area:

$$
\begin{aligned}
H_{\varphi 2}^{\mathrm{CTR}} & =\frac{q}{a^{2}} \sum_{n=1}^{\infty} \frac{\omega_{n} J_{1}\left(\chi_{n} r / a\right)}{J_{1}^{2}\left(\chi_{n}\right)} I_{2}^{\mathrm{CTR}}, \\
I_{2}^{\mathrm{CTR}} & =A^{\mathrm{CTR}} \sin \left[\omega_{0 n}^{(11)}\left(\Psi_{n} z c^{-1}-t\right)\right] \theta\left(z_{f}-z\right),
\end{aligned}
$$

where

$$
\Psi_{n}=\sqrt{1-y_{n}^{2}\left(x_{0 n}^{(11)}\right)^{-2}},
$$

$$
A^{\mathrm{CTR}}=A^{\mathrm{CR}} T, \quad T=\frac{S+1-\beta^{2}\left(\varepsilon_{0}+y_{n}^{2}\right)}{S+1-\beta^{2}\left(\varepsilon_{0}-y_{n}^{2}\right)} \frac{4 \beta^{2}\left(\varepsilon_{0}-1\right)}{2 \beta \Psi_{n}\left(1-\beta^{2}\right)\left(\varepsilon_{0}-1\right)-\left(1+\beta \Psi_{n}\right)\left[S-1-\beta^{2}\left(\varepsilon_{0}+y_{n}^{2}-2\right)\right]} .
$$

Note that the pole being considered is always situated within the interval $\tilde{\omega}_{n}^{(1)}<\omega_{0 n}^{(11)}<\omega_{\mathrm{r}}$ (Fig. 2). If $\omega_{0 n}^{(11)}>\omega_{\mathrm{n}}$, then the contribution of this pole is a propagating wave, that is, this mode is a part of the CTR. If $\omega_{0 n}^{(11)}<\omega_{n}$, then this mode exponentially decreases with the distance from the boundary and it does not transport the electromagnetic energy. One can show that the number of propagating modes (which compose the CTR) is always finite. The conditions for exciting the propagating mode with the number $n$ at $\omega_{\mathrm{r}}>\omega_{n}$ are as follows:

$\beta<\left\{\begin{array}{ll}\beta_{n} & \text { if } \beta_{n}<1 \\ 1 & \text { if } \beta_{n}>1\end{array}, \quad \beta_{n}=\left(\omega_{\mathrm{r}}^{2}-\omega_{n}^{2}\right)^{1 / 2} \omega_{\mathrm{p}}^{-1}\right.$.

The threshold value $\beta_{n}$ is explained by the total internal reflection of the CR off the boundary. This effect is observed at $\omega_{\mathrm{r}}>\omega_{n}$ and $\beta>\beta_{n}\left(\beta_{n}<1\right)$ or at $\omega_{\mathrm{r}}<\omega_{n}$. 
This limitation can also be obtained based on the Brillouin's concept, which indicates that one of the CR modes (9) consists of convergent and divergent waves: $\exp \left[ \pm \mathrm{i} \chi_{n} r a^{-1} \pm \mathrm{i} \omega_{0 n}^{(11)}\left(z c^{-1} \beta^{-1}-t\right)\right]$. The angle of incidence of these waves is less than the critical (minimum) angle for the total internal reflection when CR penetrates through the boundary. This critical angle is defined by the formula $\sin \theta_{\mathrm{C}}=\varepsilon_{1}^{-1 / 2}$. Thus, we obtain the inequality

$$
\beta \omega_{n}\left(\beta^{2} \omega_{n}^{2}+\omega_{0 n}^{(11) 2}\right)^{-1 / 2}<\left.\varepsilon_{1}^{-1 / 2}\right|_{\omega=\omega_{0 n}^{(11)}},
$$

and the solution for this result is expressed by Eq. (14).

For nondispersive dielectrics [4], the threshold $\beta_{n}$ is $\left(\varepsilon_{0}-1\right)^{-1 / 2}$, which is not dependent on the mode number. Thus, the generation of a multimode CTR occurs in a vacuum area compared with the case of a resonant medium when the CTR only has a finite number of modes.

In the vacuum area, the CTR exists in the domain

$$
z<z_{f}=c t \Psi_{n},
$$

where $\Psi_{n}$ is defined by Eq. (12). This inequality is obtained from the condition of the intersection of the pole at the transformation of the initial integration path to the steepest descent path (analogous procedure was described in [3-7]). One can show that the front of the CTR wave $z_{f}$ propagates with the group velocity of the waveguide waves $V_{g}=c \Psi_{n}$. It is dependent on the mode number and on the charge velocity $\beta$, whereas the product of the group and the phase velocities is constant $V_{g} \cdot V_{\mathrm{ph}}=c^{2}$. Analytical estimations and computations (below) indicate that the CTR of these modes comprises the main part of radiation in the vacuum area if the condition (14) is fulfilled for some mode numbers.

Analysis of the CR (9) and CTR (11) shows that the frequencies of the $\mathrm{CR}$ and CTR waves are equivalent $\omega_{\mathrm{r}} x_{0 n}^{(11)}$ [refer to (7)] which is always less than the resonance frequency $\omega_{\mathrm{r}}$. However, the wave number in the $\mathrm{z}$ direction for the $\mathrm{CR} h^{\mathrm{CR}}=\omega_{\mathrm{r}} x_{0 n}^{(11)}(c \beta)^{-1}$ differs from the wave number for the CTR $h^{\mathrm{CTR}}=\omega_{\mathrm{r}} x_{0 n}^{(11)} \Psi_{n} c^{-1}$. Some simple approximations for the CR (10) and CTR (13) amplitudes can be obtained for different velocities of $\beta$. If $\beta \ll 1$, then

$$
\begin{aligned}
A^{\mathrm{CR}} & =4 y_{n}\left(\varepsilon_{0}-1\right) \beta^{4}\left[1+O\left(\beta^{2}\right)\right], \\
A^{\mathrm{CTR}} & =8 y_{n}\left(\varepsilon_{0}-1\right) \beta^{5}\left(1-y_{n}^{2}\right)^{-1 / 2}[1+O(\beta)] .
\end{aligned}
$$

If $\beta \approx 1$ and $\beta_{n} \geq 1$, then

$$
\begin{aligned}
A^{\mathrm{CR}} & =4\left(\varepsilon_{0}-1\right)\left(y_{n}^{2}+\varepsilon_{0}-1\right)^{-3 / 2}\left[1+O\left(\gamma^{-2}\right)\right], \\
A^{\mathrm{CTR}} & =A^{\mathrm{CR}} T, \quad T \approx 2\left[1+\left(y_{n}^{2}+\varepsilon_{0}\right) \sqrt{2-y_{n}^{2}-\varepsilon_{0}}\right]^{-1},
\end{aligned}
$$

where $\gamma=\left(1-\beta^{2}\right)^{-1 / 2}$. The CTR mode amplitude may exceed the $\mathrm{CR}$ mode amplitude. A maximum increase in
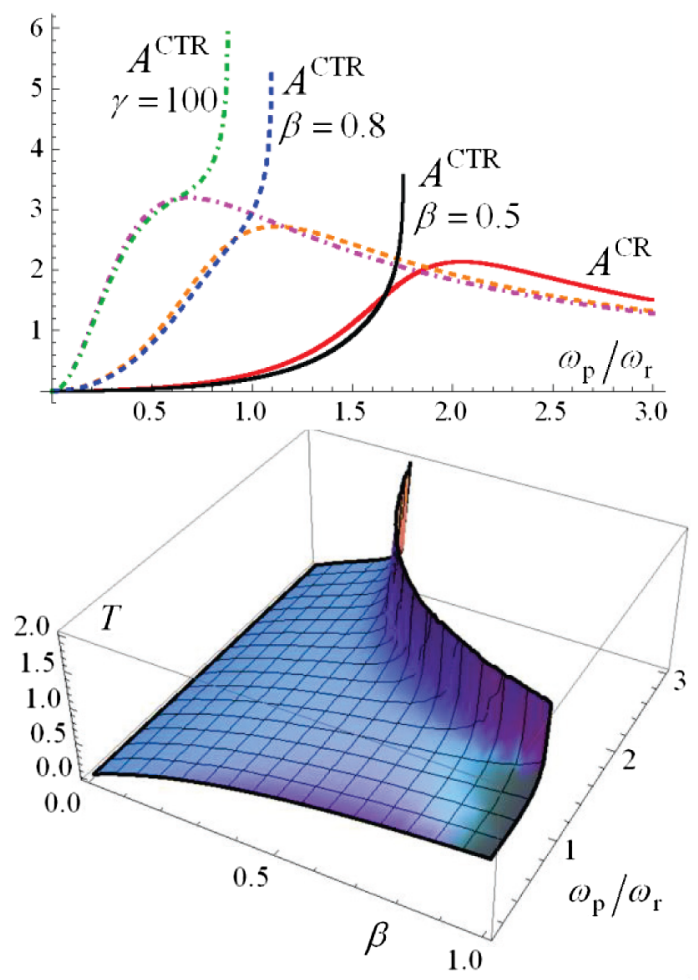

FIG. 3. The first modes of the CTR and CR amplitudes (top) and the ratio $T=A^{\mathrm{CTR}} / A^{\mathrm{CR}}$ (bottom), which is dependent on the charge velocity $\beta$ and the ratio $\omega_{\mathrm{p}} / \omega_{\mathrm{r}} ; \mathrm{n}=1, x_{\mathrm{r}}=5$.

the CTR amplitude is twice the CR amplitude. In this situation the velocity of the CTR front $V_{g} \approx c \sqrt{2-y_{n}^{2}-\varepsilon_{0}}$ also decreases. If $\beta \approx \beta_{n}<1$, then

$A^{\mathrm{CR}} \approx \frac{4\left(1-y_{n}^{2}\right)^{2}}{1+y_{n}^{4}+y_{n}^{2}\left(\varepsilon_{0}-3\right)}, \quad A^{\mathrm{CTR}} \approx 2 A^{\mathrm{CR}}, \quad V_{g} \ll c$.

The behaviors of the amplitudes of the first modes of the CTR and the CR (top) and the ratio $T=A^{\mathrm{CTR}} / A^{\mathrm{CR}}$ determined by Eq. (13) (bottom) are presented in Fig. 3 for different parameters of the problem. As can be seen, the CTR only exists at $\beta<\beta_{n}$ (note that $\beta_{n}<1$ if $\omega_{\mathrm{p}}>\sqrt{\omega_{\mathrm{r}}^{2}-\omega_{n}^{2}}$ ). A significant increase in the CTR amplitude (with the maximum value $T=2$ ) is observed for the charge velocity $\beta=\beta_{n}$. The CTR amplitude is comparable to the $\mathrm{CR}$ amplitude in an extensive range of parameters of the problem.

\section{NUMERICAL INVESTIGATION AND DISCUSSION}

The exact integral representations (3), (4) are used to calculate the free-field components. The numerical algorithm is based on a certain transformation of the initial integration path in the complex plane of $\omega$. This algorithm was previously used for the computation of the field in different dispersive unbounded [15] or semibounded [21] 
media including the case of a waveguide with semibounded cold plasma [2].

First, we rewrite the exact formulas into the integrals between the limits 0 and $+\infty$. Second, we transform the integration path into a new contour in the upper-half plane for $|z|>c t$ (before the "wave front") and into another contour in the lower-half plane for $|z|<c t$ (after the "wave front"). These new contours are presented in Fig. 2 with green dashed line and red dotted line. They should bypass all of the singularities and subsequently go parallel to the steepest descent path. Note that accurate calculations of the free-field components can be obtained by optimizing the characteristics of these new contours.

The results are presented in Fig. 4. Ammonia [22] is considered to be a typical medium; it has a resonance of $23.87 \mathrm{GHz}$ and nondimensional value $x_{\mathrm{r}}=5$ [refer to Eq. (6)] at $a \approx 1 \mathrm{~cm}$. Thus, the CTR in the vacuum area is only composed of the first mode (the remaining modes are evanescent). The critical value $\beta_{n}=1.1$, that is, CTR is generated for any charge velocity.

Figure 4 shows the component $H_{\varphi}$ of the first mode of the total field in the resonant medium and in the vacuum area for different values of velocity $\beta$ at different time moments. The forced field (8) in the medium and the analytical approximation for the CTR (11) are also presented.

As can be seen, the forced field consists of CR and a quasi-Coulomb field, which comprise the main parts of the total field in a dispersive dielectric and in a vacuum domain near the charge, respectively. If $\beta<\beta_{n}$ (14), the first mode of the CR is transmitted through the boundary and the single-mode CTR effect occurs in the vacuum area. For this situation the upper threshold for CTR in a vacuum is not important $\left(\beta_{n}>1\right)$, and CTR is generated by charges with any velocities (including the ultrarelativistic charges with $\gamma \gg 1$ ). The remaining modes of the CR are totally

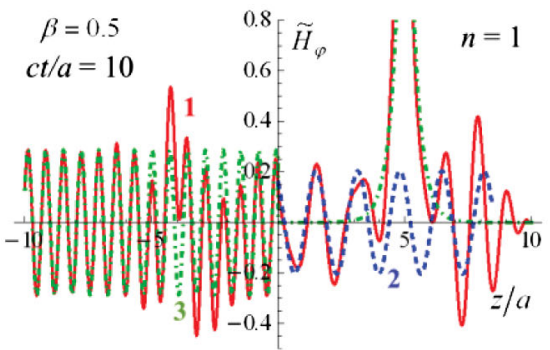

(a)

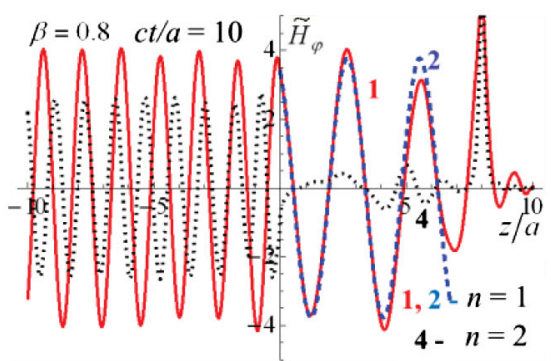

(d)

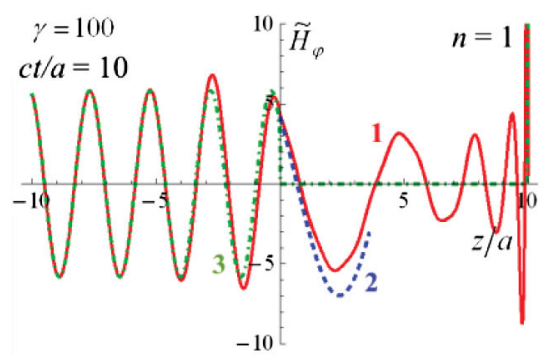

(g)

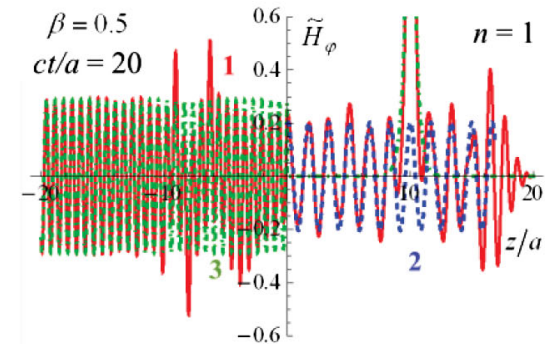

(b)

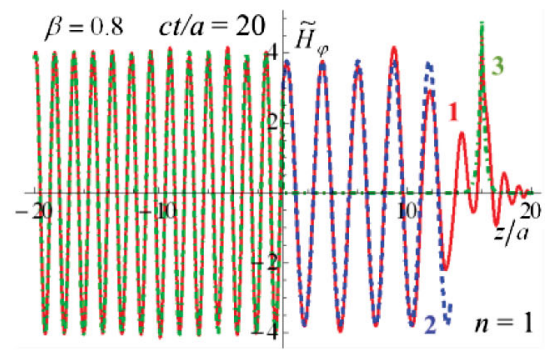

(e)

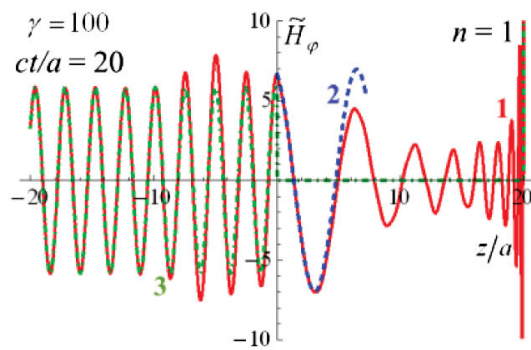

(h)

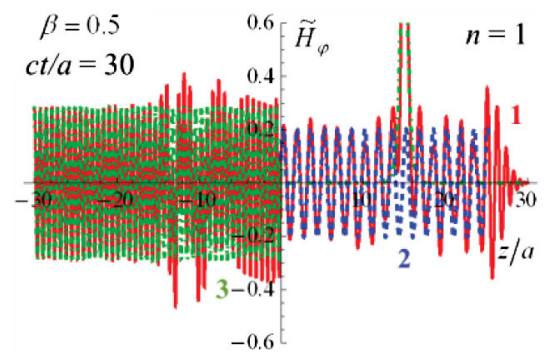

(c)

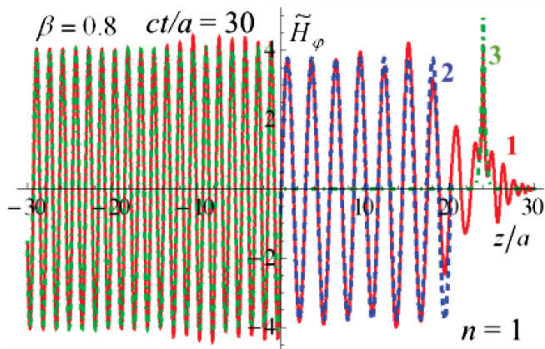

(f)

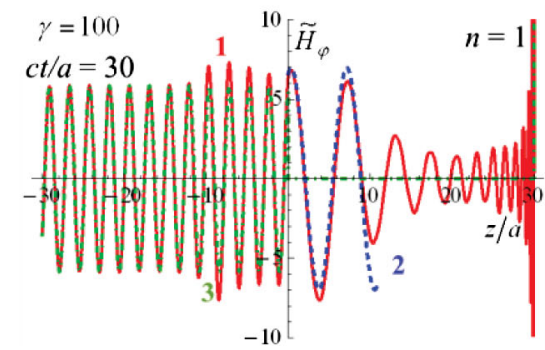

(i)

FIG. 4. Dependence of the normalized transversal component $\tilde{H}_{\varphi}=H_{\varphi} a^{2} / q$ of the $1^{s t}$ mode of the total field (solid red line 1) on the distance $z / a$ at $r=0.5$ for different values of the dimensionless time $c t / a$ and the velocity $\beta$. The dashed blue line 2 pertains to the CTR, the dot-dashed green line $\mathbf{3}$ pertains to the forced field, and the dotted black line $\mathbf{4}$ pertains to the $2^{d}$ mode of the total field (d); $\gamma=\left(1-\beta^{2}\right)^{-1 / 2}, x_{\mathrm{r}}=5, \omega_{\mathrm{p}} / \omega_{\mathrm{r}}=0.8$, and $\beta_{n}=1.1$. 
reflected off the boundary [the second mode is also shown in Fig. 4(d)].

The free field consists of the CTR and the TR in the vacuum area. The front of the TR propagates with the light velocity $c$ [2]. The computations show that the total field is approximately equal to the CTR in the domain $z<z_{f}(15)$. The dimension of the zone $z_{f}$ is on the increase in the group velocity of the waveguide waves $V_{g}=c \Psi_{n}$ (12). The CTR can be in front of the charge for the relatively small velocity of the charge motion [Fig. 4(a)-(c)]. For ultrarelativistic particles CTR and TR always occur after the charge [Fig. 4(g)-(i)]. The frequencies of the CR and CTR waves, which are the same, depend on the charge velocity and lay within the interval $12-22 \mathrm{GHz}$ for the results presented in Fig. 4. One can see that the amplitude of CTR is comparable to the amplitude of $\mathrm{CR}$ in an extensive range of parameters; it becomes slightly more $(T \approx 1.2)$ for the ultrarelativistic case. An increase in the CTR amplitude in the range $1<$ $T<2$ is possible for some parameters of the problem. Note that the CR amplitude might have maximum magnitude up to $500 \mathrm{KV} / \mathrm{m}$ for an electron bunch with $|q|=1 \mathrm{nC}$ [4].

Phenomena under consideration can also take place in $\mathrm{THz}$ frequency range if the medium has a corresponding resonance. As an example, we can point out Cesium Bromide ( $\mathrm{CsBr})$ which has several resonances [23]: one of them lies at $2.2 \mathrm{THz}$, and the others have much more frequencies. Nondimensional value $x_{\mathrm{r}}$ (6) depends on a waveguide radius $a$; for example, $x_{\mathrm{r}}=230$ for $a=5 \mathrm{~mm}$. For these parameters, the CTR consist of 73 modes in the vacuum area, and of 62 modes for an ultrarelativistic bunch (limit value $\beta_{n}>1$ for $n<63, \omega_{\mathrm{p}}=0.52 \omega_{\mathrm{r}}$ ). The frequencies of the CTR depend on the charge velocity and lay within the interval $0.04-2.1 \mathrm{THz}$.

Note that, in recent years, waveguides with radiuses of the order of $100-1000 \mu \mathrm{m}$ are actively used for generation of Cherenkov radiation [10]. If the filling material is $\mathrm{CsBr}$ and $a=400 \mu \mathrm{m}$, then $x_{\mathrm{r}}=18.4$, the number of the CTR modes is 6 , and $\beta_{n}>1$ for $n<6$. Single mode condition of the CTR can be reached for waveguide with radius being about $100 \mu \mathrm{m}$. Thus, phenomena discussed here can occur in $\mathrm{THz}$ range as well as in $\mathrm{GHz}$ one.

\section{CONCLUSION}

The electromagnetic field of a charged particle that moves in a circular waveguide and intersects the boundary between a resonant medium and a vacuum was considered. The analysis of the field components was analytically and numerically performed. The analytical investigation was based on the steepest descent method of the complex variable function theory. The algorithm for the computation was also presented.

The analytical and numerical investigations show that the CTR in the vacuum area always consists of a finite number of propagating modes (compared with the case of nondispersive dielectric when the CTR has an infinite number of modes). The other modes of CR are totally reflected off the boundary and penetrate in the vacuum area in the form of evanescent waves. We can select the parameters of a problem to ensure that the CTR only has the first waveguide mode, that is, we can obtain monochromatic radiation.

The CTR can be the main part of the wave field in the vacuum area of the waveguide for the previously described conditions. The amplitudes of the CTR modes in a vacuum may exceed the amplitudes of the CR modes in the resonant medium. The CTR effect in the considered situation can be used for generation of intense single-mode monochromatic radiation in the vacuum part of the waveguide.

\section{ACKNOWLEDGMENTS}

This research was supported by the Russian Foundation for Basic Research (Grant No. 15-02-03913a).

[1] K. A. Barsukov, Transition radiation in a waveguide, Zh. Eksp. Teor. Fiz. 37, 1106 (1959) [Sov. Phys. JETP 37, 787 (1960)].

[2] T. Y. Alekhina and A. V. Tyukhtin, Electromagnetic field of a charge intersecting a cold plasma boundary in a waveguide, Phys. Rev. E 83, 066401 (2011).

[3] T. Y. Alekhina and A. V. Tyukhtin, Radiation of a charge in a waveguide with a boundary between two dielectrics, J. Phys. Conf. Ser. 357, 012010 (2012).

[4] T. Y. Alekhina and A. V. Tyukhtin, Cherenkov-transition radiation in a waveguide with a dielectric-vacuum boundary, Phys. Rev. ST Accel. Beams 15, 091302 (2012).

[5] T. Y. Alekhina and A. V. Tyukhtin, Self-acceleration of a charge intersecting a boundary surface in a waveguide, Phys. Rev. ST Accel. Beams 16, 081301 (2013).

[6] T. Y. Alekhina and A. V. Tyukhtin, Electromagnetic field of a bunch intersecting a vacuum gap in a dielectric loaded waveguide, Phys. Rev. ST Accel. Beams 17, 071302 (2014).

[7] T. Y. Alekhina and A. V. Tyukhtin, Electromagnetic field of a bunch intersecting a dielectric plate in a waveguide, J. Phys. Conf. Ser. 517, 012012 (2014).

[8] J. G. Power, M. E. Conde, W. Gai, A. Kanareykin, R. Konecny, and P. Schoessow, Measurements of the longitudinal wakefields in a multimode, dielectric wakefield accelerator driven by a train of electron bunches, Phys. Rev. ST Accel. Beams 3, 101302 (2000).

[9] A. Kanareykin, Cherenkov radiation and dielectric based accelerating structures: Wakefield generation, power extraction and energy transfer efficiency, J. Phys. Conf. Ser. 236, 012032 (2010).

[10] A. M. Cook, R. Tikhoplav, S. Y. Tochitsky, G. Travish, O. B. Williams, and J. B. Rosenzweig, Observation of Narrow-Band Terahertz Coherent Cherenkov Radiation from a Cylindrical Dielectric-Lined Waveguide, Phys. Rev. Lett. 103, 095003 (2009). 
[11] G. Andonian, O. Williams, X. Wei, P. Niknejadi, E. Hemsing, J. B. Rosenzweig, P. Muggli, M. Babzien, M. Fedurin, K. Kusche, R. Malone, and V. Yakimenko, Resonant excitation of coherent Cerenkov radiation in dielectric lined waveguides, Appl. Phys. Lett. 98, 202901 (2011).

[12] S. Antipov, C. Jing, A. Kanareykin, J. E. Butler, V. Yakimenko, M. Fedurin, K. Kusche, and W. Gai, Experimental demonstration of wakefield effects in a THz planar diamond accelerating structure, Appl. Phys. Lett. 100, 132910 (2012).

[13] G. N. Afanasiev and V. G. Kartavenko, Radiation of a point charge uniformly moving in a dielectric medium, J. Phys. D 31, 2760 (1998).

[14] G. N. Afanasiev, V. G. Kartavenko, and E. N. Magar, Vavilov-Cherenkov radiation in dispersive medium, Physica B (Amsterdam) 269, 95 (1999).

[15] A. V. Tyukhtin and S. N. Galyamin, Vavilov-Cherenkov radiation in passive and active media with complex resonant dispersion, Phys. Rev. E 77, 066606 (2008).
[16] B. M. Bolotovskiy, Theory of Cherenkov radiation, Phys. Usp. 75, 295 (1961).

[17] A. V. Tyukhtin, Radiation of a charge moving in a waveguide filled with a dielectric medium possessing resonance dispersion, Tech. Phys. Lett. 30, 605 (2004).

[18] A. V. Tyukhtin, Radiation of a charge moving in a waveguide with a resonantly dispersive dielectric layer, Tech. Phys. Lett. 31, 150 (2005).

[19] V. L. Ginzburg and V. N. Tsytovich, Transition Radiation and Transition Scattering (Hilger, London, 1990), p. 445.

[20] B. Felsen and N. Marcuvitz, Radiation and Scaterring of Waves (Wiley Interscience, New York, 2003), p. 888.

[21] S. N. Galyamin and A. V. Tyukhtin, Electromagnetic field of a charge traveling into an anisotropic medium, Phys. Rev. E 84, 056608 (2011).

[22] L. Schăchter, Hybrid Čerenkov mode in a resonant medium, Phys. Rev. E 62, 1252 (2000).

[23] H. H. Li, Refractive index of alkali halides and its wavelength and temperature derivatives, J. Phys. Chem. Ref. Data 5, 329 (1976). 